\title{
From diabetes to cancer: New applications for targeting AMPK in the clinical setting
}

\section{Carly Barron}

\author{
Brock University
}

The emergence of $5^{\prime}$ adenosine monophosphate-activated protein kinase (AMPK) as a target for cancer was unexpected and it has subsequently proven to be a small protein with big possibilities. Although AMPK is a well known indirect target of antidiabetic drugs, its potential as a cell proliferation suppressor has only recently been investigated. A growing body of preclinical and clinical research suggests activating AMPK may be the future direction in preventative and therapeutic cancer strategies.

AMPK is a major regulator of metabolism in eukaryotic cells ${ }^{1}$. It is a serine/threonine kinase activated by metabolic stressors which deplete ATP and increase AMP levels ${ }^{2}$. Once activated, AMPK can restore energy homeostasis by suppressing enzymes involved in ATP consumption and increasing ATP production ${ }^{2}$. Patients with disorders in which AMPK activity is decreased, including metabolic syndrome and diabetes, have an increased risk of developing various cancers ${ }^{3}$.

AMPK has been shown to suppress cell proliferation in non-malignant and tumor cells ${ }^{4}$. This activity may be explained by the tumor suppressor genes that lie within the AMPK pathway including LKB $1^{4}$. The mammalian target of rapamycin (mTOR) pathway is a key regulator of protein translation/synthesis; AMPK activation inhibits mTOR signaling limiting the amount of protein cells that have to grow and divide ${ }^{4}$. These observations suggest agents that activate AMPK may be useful to prevent tumor development and growth. Metformin is the most widely prescribed oral hypoglycemic drug. It is believed to have antitumorigenic effects that are independent of its hypoglycemic effects and has received attention as a novel-anticancer agent ${ }^{5}$. Studies have shown the mechanism by which metformin can inhibit cancer cell growth is mediated mainly by AMPK ${ }^{1}$.
The effects of metformin on cancer mortality have been suggested to be dose-dependent ${ }^{7}$. Furthermore, epidemiological studies support the notion that metformin has anticancer properties as diabetics receiving this drug display a dose-dependent reduced risk of cancer $^{6}$. But can metformin be used to treat existing cancers? Several clinical trials are currently underway to investigate the safety and efficacy of metformin in patients with breast, pancreatic and prostate cancer ${ }^{1}$. Phase II and III trials will compare invasive-disease free survival in patients treated with metformin versus a placebo or standard treatment ${ }^{1}$.

In addition to possibly enhancing chemotherapy, activation of AMPK sensitizes cancer cells to the cytotoxic effects of ionizing radiation $(\mathrm{IR})^{8}$. It was recently reported that AMPK is activated by IR in epithelial cancer cells and targeting AMPK pharmacologically enhanced the IR response ${ }^{8}$. Targeting AMPK to enhance the effects of IR may be especially beneficial for treating lung and prostate cancers in which even high doses of radiotherapy show limited efficacy ${ }^{9}$.

Specific activators of AMPK that do not alter cellular AMP levels are currently under investigation ${ }^{10}$. Direct activators, such as A-769662, act more potently and effectively than metformin and in a greater range of tissues $^{11}$. A direct AMPK activator with good bioavailability would be ideal for clinical use to prevent undesired nonspecific effects.

So is it time for the clinical development of AMPK activators for the prevention and treatment of cancer? To put it simply, not quite. Although the in vitro and in vivo evidence demonstrating a link between AMPK and cancer is compelling, the epidemiological evidence is limited by confounders and the study designs used. Only two of the ongoing clinical trials studying metformin and 
cancer set out to determine the maximum tolerated dose of metformin in study patients. There is sufficient rationale to study AMPK activators in the clinical setting; however, a few issues remain to be addressed.

The safety data in cancer patients should be established. It should be determined whether there are consequences of tampering with glucose metabolism in non-diabetic subjects. The current clinical trials using metformin are short-term studies; therefore, the long-term effects of taking metformin in these individuals should be investigated. Clinical trials should provide vital information about the magnitude of the effect of metformin in non-diabetic compared to diabetic patients, since hyperinsulinemia in diabetes is considered a risk factor for malignancies ${ }^{5}$. The minimum dose of AMPK activators to achieve an antiproliferative effect and the maximum dose tolerated in cancer patients needs to be established. Increased AMPK activity has previously been shown to affect cardiac function ${ }^{12}$, therefore, it is possible consequences of systemic AMPK activators may occur at the maximum dosage.

Increased mTOR activation and decreased AMPK activation have been suggested as predictive biomarkers of the efficacy of these drugs ${ }^{13}$. Subjects with indication of increased mTOR activity, such as S6K phosphorylation, would benefit from AMPK-mediated inhibition of this pathway due to prevention of cell growth and proliferation. There is debate whether LKB1 has to be intact for activation of AMPK; therefore, some tumors may not respond to this type of treatment ${ }^{11}$. Future identification of patients likely to respond to AMPK activators using these suggested biomarkers will improve the success of clinical trials.

Finally, identifying treatments to combine with AMPK activators may be most effective in the clinical setting information which can be gained from clinical and retrospective studies. As well, using agents to activate AMPK prior to IR may provide the maximum benefit for patients receiving radiotherapy.

As new information comes forward supporting the link between AMPK and cancer, it is critical to understand the mechanisms by which it suppresses cell proliferation, however, the transition of targeting AMPK from "bench to bedside" is certainly on its way.

\section{References}

1. Kourelis TV, Siegel RD: Metformin and cancer: New applications for an old drug. Med Oncol. 2011 [Epub ahead of print].
2. Zadra G, Priolo C, Patnaik A, Loda M: New Strategies in Prostate Cancer: Targeting Lipogenic Pathways and the Energy Sensor AMPK. Clin Cancer Res. 16(13):33223328, 2010.

3. Luo Z, Saha AK, Xiang X, Ruderman NB. AMPK, the metabolic syndrome and cancer. Trends Pharmacol Sci. 26:69-72, 2005.

4. Motoshima H, Goldstein BJ, Igata M, Araki E: AMPK and cell proliferation ---AMPK as a therapeutic target for atherosclerosis and cancer. J Physiol. 574:63-71, 2006.

5. Jalving M, Gietema JA, Lefrandt JD, de Jong S, Reyners AK, Gans RO, de Vries EG: Metformin: Taking away the candy for cancer? Eur J Cancer. 46:2369-2380, 2010.

6. Evans JM, Donnelly LA, Emslie-Smith AM, Alessi DR, Morris AD. Metformin and reduced risk of cancer in diabetic patients. BMJ. 330:1304-1305, 2005.

7. Landman GW, Kleefstra $\mathrm{N}$, van Hateren $\mathrm{KJ}$, et al. Metformin associated with lower cancer mortality in type 2 diabetes: ZODIAC-16. Diabetes Care, 33: 322-326, 2010.

8 Sanli T, Rashid A, Liu C, Harding S, Bristow RG, Cutz JC, Singh G, Wright $\mathrm{J}$, Tsakiridis T: Ionizing radiation activates AMP-activated kinase (AMPK): A target for radiosensitization of human cancer cells. Int $J$ Radiat Oncol Biol Phys. 78:221-229, 2010

9. MacRae R, Choy H. Concurrent chemoradiotherapy for inoperable stage III non-small -cell lung cancer. Curr Oncol Rep. 5:313-317, 2003

10. Cool B, Zinker B, Chiou W, et al. Identification and characterization of a small molecule AMPK activator that treats key components of type 2 diabetes and the metabolic syndrome. Cell Metab. 3:403-416, 2006.

11. van Veelen W, Korsse SE, van de Laar L, Peppelenbosch MP: The long and winding road to rational treatment of cancer associated with LKB1/AMPK/TSC/mTORC1 signaling. Oncogene. 2011[Epub ahead of print].

12. Arad M, Seidman C.E., Seidman J.G. AMP-activated protein kinase in the heart: role during health and disease. Circ Res. 100:474-488, 2007.

13. Fay JR, Steele V, Crowell JA: Energy homeostasis and cancer prevention: The AMP-activated protein kinase. Cancer Prev Res. 2:301-309, 2009

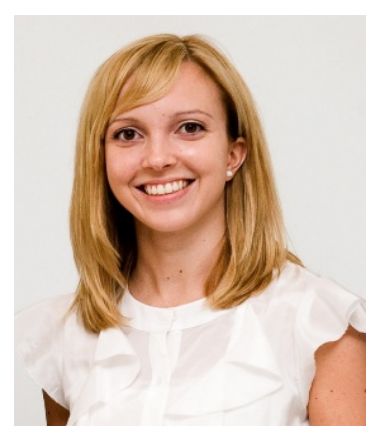

\section{Author Profile}

Carly Barron is currently pursuing a MSc degree in Applied Health Sciences at Brock University. Her main research interests include the molecular and cellular biology of mammalian glucose transporter (GLUT) proteins and their role in glucose homeostasis and disease. 